\title{
Erratum to: Recurrent malignant melanoma of the palate successfully treated by gamma knife radiosurgery
}

\author{
Shin-ichi Yamada - Souichi Yanamoto - Akiko Kawakita • \\ Goro Kawasaki · Shuichi Fujita • Tohru Ikeda • \\ Takemitsu Matsuo • Masahiro Umeda
}

Published online: 27 March 2013

(C) Japanese Society for Oral and Maxillofacial Radiology and Springer Japan 2013

\section{Erratum to: Oral Radiol}

DOI 10.1007/s11282-012-0120-2

Unfortunately, Figs. 2c and 8a have been published incorrectly as a result of an error during the typesetting process. The correct version of the figures follows.

The online version of the original article can be found under doi:10.1007/s11282-012-0120-2.

S. Yamada $(\bowtie) \cdot$ S. Yanamoto · A. Kawakita $\cdot$ G. Kawasaki .

M. Umeda

Department of Clinical Oral Oncology, Nagasaki University

Graduate School of Biomedical Sciences, 1-7-1 Sakamoto,

Nagasaki 852-8588, Japan

e-mail: shinshin@nagasaki-u.ac.jp

S. Fujita $\cdot$ T. Ikeda

Department of Oral Pathology and Bone Metabolism,

Unit of Translational Medicine, Course of Medical

and Dental Sciences, Nagasaki University Graduate

School of Biomedical Sciences, Nagasaki, Japan

T. Matsuo

Department of Oral and Maxillofacial Surgery,

Omura Municipal Hospital, Omura, Japan 


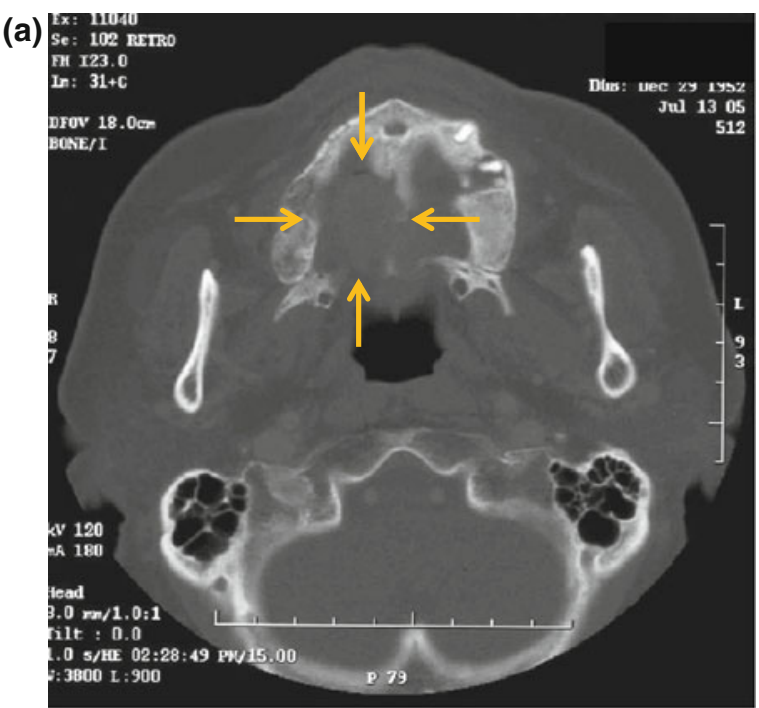

(b)

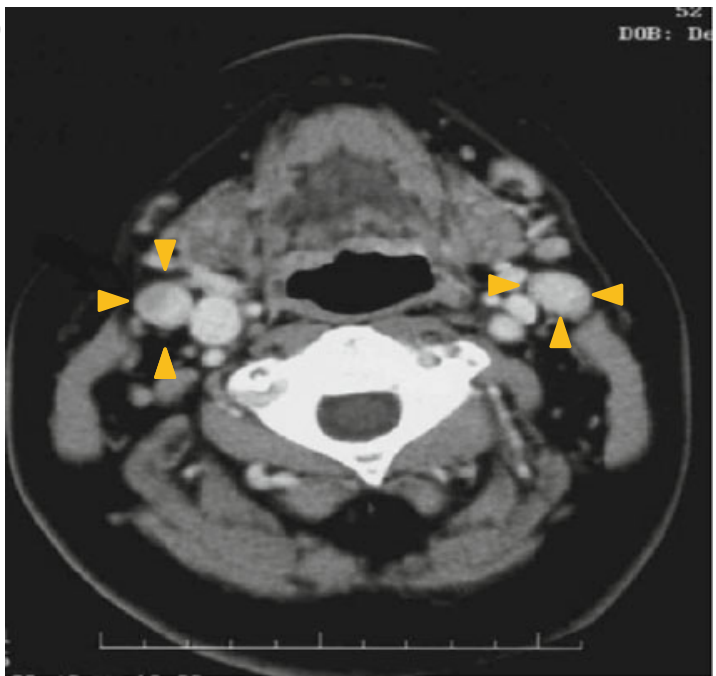

(c) ISE/M

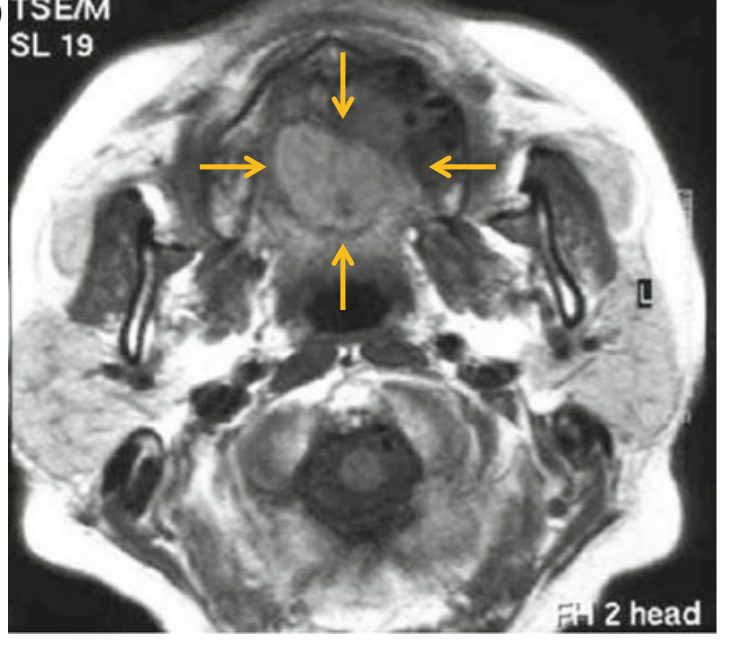

Fig. 2 a Axial enhanced CT scan revealing bone destruction in the right hard palate (arrows) (window width, 3800; window level, 900). b Axial enhanced CT scan revealing enlarged lymph nodes in the bilateral level IIA regions (arrowheads) (window width, 250; window level, 50). c Axial enhanced MR T1-weighted image revealing an enhanced mass lesion in the right hard palate (arrows)
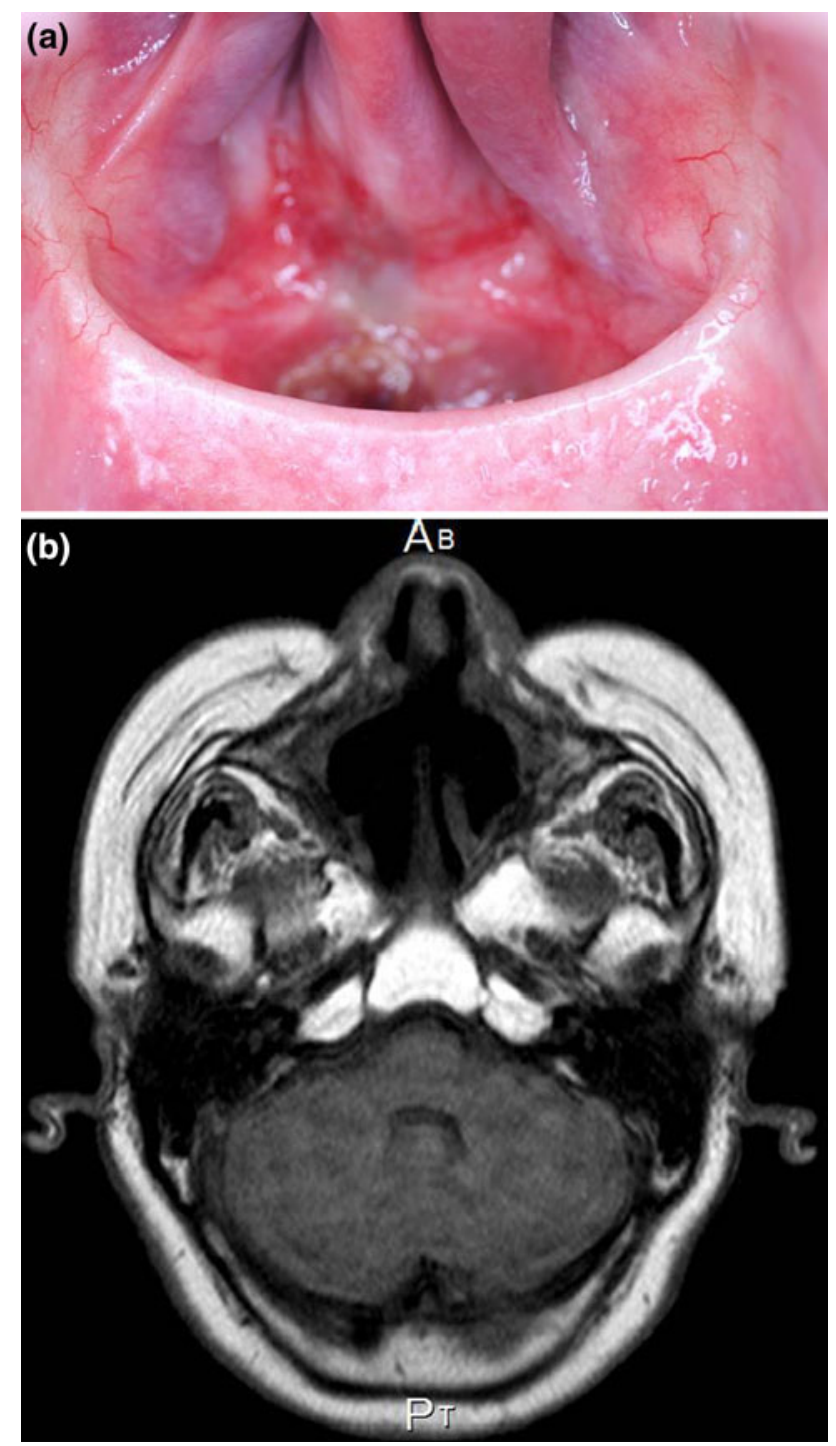

Fig. 8 a Photograph showing no tumor recurrence in the nasal septum at 57 months after GKS. b Axial enhanced MR T1-weighted image revealing no recurrence in the clivus 